\title{
OPEN Skin bacteria of rainbow trout antagonistic to the fish pathogen Flavobacterium psychrophilum
}

\begin{abstract}
Mio Takeuchi ${ }^{1 \bowtie}$, Erina Fujiwara-Nagata², Taiki Katayama ${ }^{3}$ \& Hiroaki Suetake ${ }^{4}$
Rainbow trout fry syndrome (RTFS) and bacterial coldwater disease (BCWD) is a globally distributed freshwater fish disease caused by Flavobacterium psychrophilum. In spite of its importance, an effective vaccine is not still available. Manipulation of the microbiome of skin, which is a primary infection gate for pathogens, could be a novel countermeasure. For example, increasing the abundance of specific antagonistic bacteria against pathogens in fish skin might be effective to prevent fish disease. Here, we combined cultivation with 16S rRNA gene amplicon sequencing to obtain insight into the skin microbiome of the rainbow trout (Oncorhynchus mykiss) and searched for skin bacteria antagonistic to F. psychrophilum. By using multiple culture media, we obtained 174 isolates spanning 18 genera. Among them, Bosea sp. OX14 and Flavobacterium sp. GL7 respectively inhibited the growth of $F$. psychrophilum KU190628-78 and NCIMB 1947 ${ }^{\top}$, and produced antagonistic compounds of $<3 \mathrm{kDa}$ in size. Sequences related to our isolates comprised $4.95 \%$ of skin microbial communities, and those related to strains OX14 and GL7 respectively comprised $1.60 \%$ and $0.17 \%$ of the skin microbiome. Comparisons with previously published microbiome data detected sequences related to strains OX14 and GL7 in skin of other rainbow trout and Atlantic salmon.
\end{abstract}

An increasing global population has caused a concomitant increased demand for food. Aquaculture is the world's fastest growing food production sector ${ }^{1}$, partly fulfilling the food demand. Infectious diseases are among the most pressing concerns in aquaculture development. For example, both rainbow trout fry syndrome (RTFS) and bacterial coldwater disease (BCWD) caused by Flavobacterium psychrophilum ${ }^{2,3}$ have become major causes of economic losses in salmonid fish such as rainbow trout (Oncorhynchus mykiss), coho salmon (O. kisutsh), and ayu (Plecoglossus altivelis) aquaculture worldwide. Fish diseases are usually controlled by antibiotics ${ }^{4}$, but antimicrobial resistance is a threat to the treatment worldwide. In fact, the World Health Organization (WHO) included antimicrobial resistance as one of the top 10 threats to global health ${ }^{5}$. Hence, a "One Health" approach, including aquaculture, is required to tackle antimicrobial resistance ${ }^{6}$. Vaccination can also prevent many bacterial diseases of fish. However, many problems need to be overcome before an effective vaccine for F. psychrophilum can become commercially available ${ }^{7}$.

Biological control is a possible alternative to antibiotics or vaccines ${ }^{8}$. Carnobacterium sp. $^{9}$, Pseudomonas sp. ${ }^{10-13}$ and others ${ }^{14-17}$ have been identified as antagonistic against $F$. psychrophilum. Thus, these bacteria have been applied as functional feeds based on the notion that they would function in the fish gut ${ }^{18}$. The survival of rainbow trout fed with antagonistic strains and challenged with $F$. psychrophilum increased to some extent ${ }^{10,11,17}$ (relative percent survival values ranged from 28 to 58\%). However, the practical application of this strategy has not yet proven successful. Recently, Nakashima et al. ${ }^{19}$ found that microbes in the digesta of ray-finned fish are separated from the epithelium by chitinous membranes that are not found in mammals. Thus, the effects of probiotics targeting the gut microbiome of fish may differ from that in mammals.

Skin damage is considered to create new portals for F. psychrophilum entry ${ }^{20}$. Therefore, manipulation of the skin microbiome, such as increasing the abundance of specific antagonistic bacteria against pathogens might be effective. Such bacteria should be residing on the skin mucus rather than the gut of fish. Boutin et al. ${ }^{15}$ and De la Fuente et al. ${ }^{16}$ isolated skin bacteria and searched for antagonists to F. psychrophilum. However, the isolated strains represented only $0.03 \%-1.8 \%$ of the total microbiota in the skin mucus ${ }^{15}$, or their population was not

\footnotetext{
${ }^{1}$ Biomedical Research Institute, National Institute of Advanced Industrial Science and Technology (AIST), 1-8-31 Midorigaoka, Ikeda, Osaka 563-8577, Japan. ${ }^{2}$ Faculty of Agriculture, Kindai University, 3327-204 Nakamachi, Nara, Nara 631-8505, Japan. ${ }^{3}$ Institute for Geo-Resources and Environments, National Institute of Advanced Industrial Science and Technology (AIST), 1-1-1 Higashi, Tsukuba, Ibaraki 305-8567, Japan. ${ }^{4}$ Faculty of Marine Science and Technology, Fukui Prefectural University, 1-1 Gakuen-cho, Obama, Fukui 917-0003, Japan. ${ }^{\square}$ email: takeuchi-mio@aist.go.jp
} 


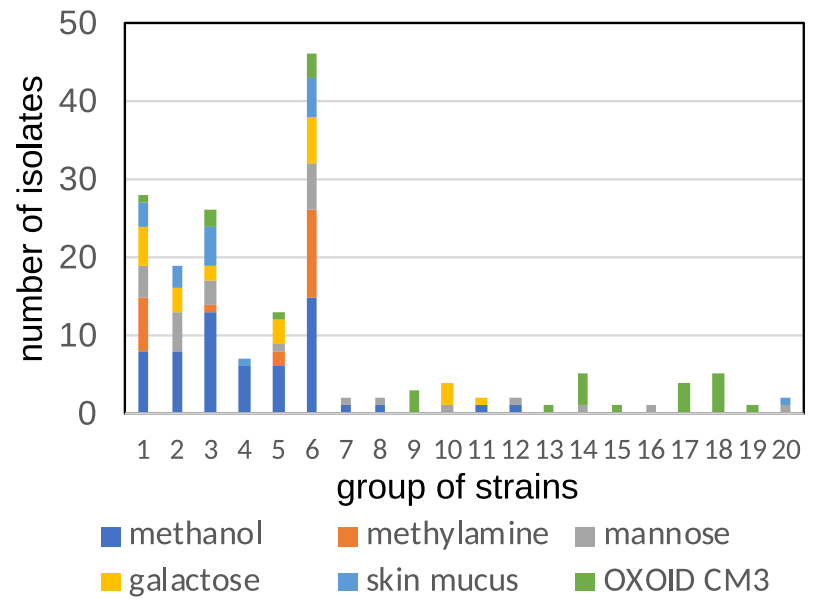

Figure 1. Numbers of isolates classified for strain group, composition of culture media.

determined ${ }^{16}$. Recently, culturomics approach is used to understand gut microbiome of human ${ }^{21}$. Similarly, more effort should be made to isolate diverse bacteria from fish skin for further understanding of skin microbiome and the screening of antagonistic bacteria.

The present study aimed to analyze the skin microbiome of rainbow trout in order to culture a range of diverse bacteria for screening their antagonistic properties. Previous studies of isolating bacteria from fish skin have used only one or two complex media ${ }^{15,22-30}$. Here, we used 10 culture media containing different carbon sources or solidifying agents to isolate more diverse bacteria from the skin mucus of the rainbow trout. We then evaluated the antagonistic ability of the isolates against four strains of F. psychrophilum. The fraction of isolated strains in microbial communities of skin, gill, and the gut of rainbow trout, and surrounding water was estimated by high throughput sequencing analysis. We also compared our findings with published microbiome data of fish $\operatorname{skin}^{29,31,32}$ to see whether similar sequences to the cultured isolates were found previously.

\section{Results}

Strains isolated from skin mucus. We used culture media containing 6 different carbon sources. Sugars were included because lectin, a major component of fish mucus, binds various sugars ${ }^{33}$. Methanol and methylamine were included to isolate methylotrophs that was dominant in the skin of brook charr and is considered to compete with F. psychrophilum ${ }^{34}$. We obtained 174 isolates in total (Supplementary Table S1 online), and sequenced the 16S rRNA gene of the isolated strains. Based on the sequence identity of $>99 \%$ (considered to belong to the same species), the isolates were assigned to 20 groups spanning 18 genera and five phyla. Previous reports $^{35,36}$ showed that closely related strains (including those shared $>99.2 \%$ of $16 \mathrm{~S}$ rRNA gene similarity ${ }^{36}$ ) similarly exhibited antagonistic activities. In this study, a representative strain was selected from each group assuming that strains of each group exhibit similar antagonistic characteristics. Six of the 20 groups contained isolates that were retrieved only from OXOID CM3 medium and nine that were retrieved from media other than OXOID CM3 (Fig. 1). Group No. 6 represented by strain GL29 was the dominant and this group was obtained by all of the culture medium used (Fig. 1).

Representative strains in each group (Table 1) were further sequenced to obtain the almost complete $16 \mathrm{~S}$ rRNA gene sequence (from 1392 to $1475 \mathrm{bp}$ ). These representative strains were used for further analyses. Strains OX11, OX32, and MN7 were closely related to possible pathogenic bacteria (Pseudomonas fluorescens, Bacillus cereus, and Aeromonas caviae, respectively) and were excluded from further analyses because it will not be practical to use these strains in future biological treatment (Table 1).

Growth of the isolates on FLP, OXOID CM3, LB, and galactose containing medium. In order to assess whether our culture strategy was effective in culturing diverse skin bacteria, we examined the ability of the isolates to grow in four media. Strains TLA9, OX16, and OX5 could not grow in galactose containing medium, but thrived in OXOID CM3 and LB media (Supplementary Table S2 online). In contrast, strains AG1, MN22, and AG5 grew in galactose containing medium, but not in OXOID CM3 and LB media. All representative strains grew in FLP medium.

Screening of antagonistic activity against $\boldsymbol{F}$. psychrophilum. Culture supernatant of the 17 representative strains were added to the FLP medium inoculated with F. psychrophilum strains, and the growth of F. psychrophilum strains were monitored. Nine out of the 17 strains exerted antagonistic action against at least one F. psychrophilum strain (Supplementary Fig. S1 online, Table 1). Among the nine antagonistic strains, OX14 and GL7 inhibited the growth of F. psychrophilum KU190628-78 and NCIMB $1947^{\mathrm{T}}$ to $<60 \%$, respectively (Supplementary Fig. S1 online). The antagonistic activities of these two strains were also confirmed using the 


\begin{tabular}{|l|l|l|l|l|l|l|}
\hline Group no & Representative strain & Closest species & Phylum & Sequence length & Identity (\%) & Antagonistic \\
\hline 1 & AG1 & Cellvibrio fibrivorans & Proteobacteria & 1440 & 99.0 & \\
\hline 2 & MN22 & Cellvibrio gandavensis & Proteobacteria & 1475 & 98.9 & \\
\hline 3 & TLA9 & Rheinheimera texasensis & Proteobacteria & 1424 & 99.7 & + \\
\hline 4 & TLA11 & Duganella ginsengisoli & Proteobacteria & 1454 & 98.4 & + \\
\hline 5 & AG5 & $\begin{array}{l}\text { Pseudaeromonas pectini- } \\
\text { lytica }\end{array}$ & Proteobacteria & 1441 & 98.8 & \\
\hline 6 & GL29 & Acinetobacter tjernbergiae & Proteobacteria & 1439 & 99.9 & + \\
\hline 7 & TLA8 & Rhodococcus qingshengii & Actinobacteriota & 1420 & 99.9 & + \\
\hline 8 & MN10 & Deinococcus aquaticus & Deinococcota & 1402 & 99.4 & + \\
\hline 9 & OX16 & Deefgea chitinilytica & Proteobacteria & 1432 & 100.0 & + \\
\hline 10 & GL27 & Rhodoferax ferrireducens & Proteobacteria & 1441 & 98.1 & \\
\hline 11 & GL7 & Flavobacterium tructae & Bacteroidota & 1411 & 98.9 & + \\
\hline 12 & MN5 & Flavobacterium succinicans & Bacteroidota & 1429 & 98.4 & \\
\hline 13 & OX15 & Pelomonas saccharophila & Proteobacteria & 1432 & 98.6 & + \\
\hline 14 & OX14 & Bosea lupine & Proteobacteria & 1392 & 99.6 & + \\
\hline 15 & OX5 & Vogesella perlucida & Proteobacteria & 1443 & 99.9 & \\
\hline 16 & MN24 & Hydrogenophaga palleronii & Proteobacteria & 1439 & 98.7 & \\
\hline 17 & OX13 & $\begin{array}{l}\text { Paenisporosarcina quisquil- } \\
\text { iarum }\end{array}$ & Firmicutes & 1460 & 100.0 & \\
\hline 18 & OX11 & Pseudomonas fluorecens & Proteobacteria & 1430 & 98.9 & NE \\
\hline 19 & OX32 & Bacillus cereus & Firmicutes & 1439 & 100.0 & NE \\
\hline 20 & MN7 & Proteobacteria & 1434 & 100.0 & NE \\
\hline
\end{tabular}

Table 1. Representative strains and closest species. Identity (\%), 16S rRNA gene similarity with closest species; NE, not evaluated; Sequence length, length of $16 \mathrm{~S}$ rRNA gene. NE, not evaluated; Sequence length, length of $16 \mathrm{~S}$ rRNA gene. +, postitive antagonistic action against at least one strain of F. psychrophilum. * Strain MN7 was also close to Aeromonas caviae at sequence identity of $99.9 \%$.

(a)

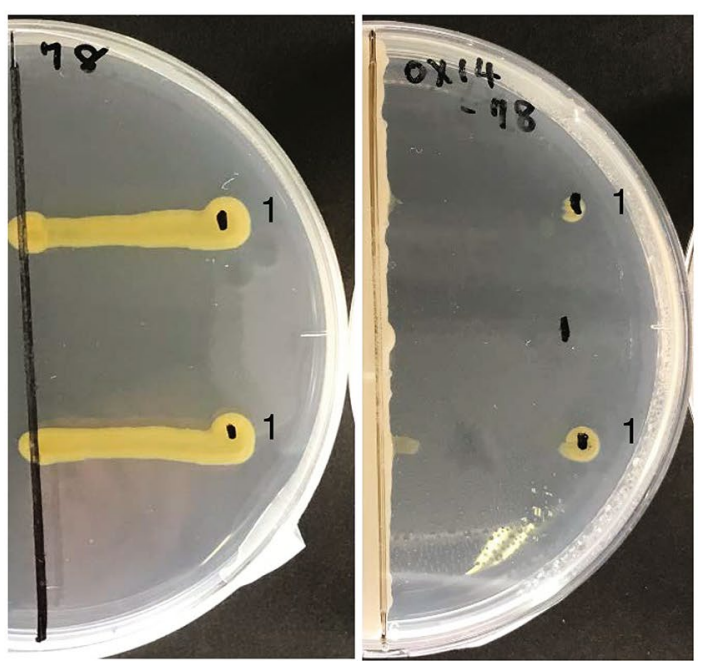

(b)

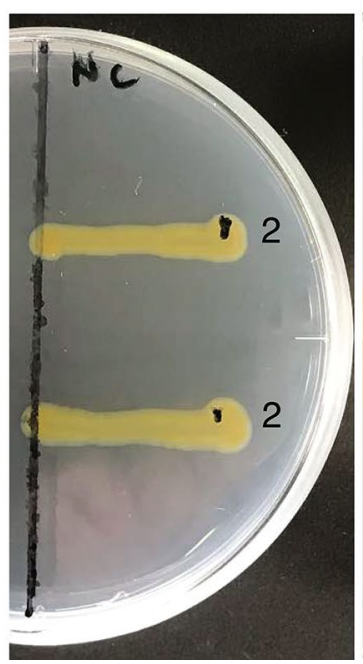

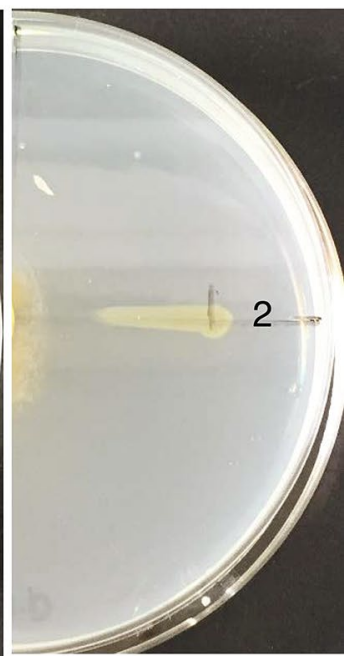

Figure 2. Cross-streaking method. The vertical line (a) and circle (b) in the right image is (a) strain OX14 and (b) GL7, and the lines marked with numerical digits are 1: F. psychrophilum KU190628-78 and 2: F. psychrophilum NCIMB 1947T. Left images are positive controls without antagonistic strains.

cross-streaking method (Fig. 2). Therefore, we further characterized antagonistic activity of strain OX14 against F. psychrophilum KU190628-78 and that of strain GL7 against F. psychrophilum NCIMB $1947^{\mathrm{T}}$.

Characteristics of antagonistic actions of strains OX14 and GL7 against F. psychrophilum. The $\mathrm{pH}$ of the culture supernatant of strain OX14 and GL7 were 7.6 and 7.2, respectively, and thus, the effect of $\mathrm{pH}$ change on the growth of $F$. psychrophilum strains was negligible. The CAS assay of siderophores was positive for strain GL7 as indicated by the color change of the medium, and weakly positive for strain OX14, suggesting the 
(a)

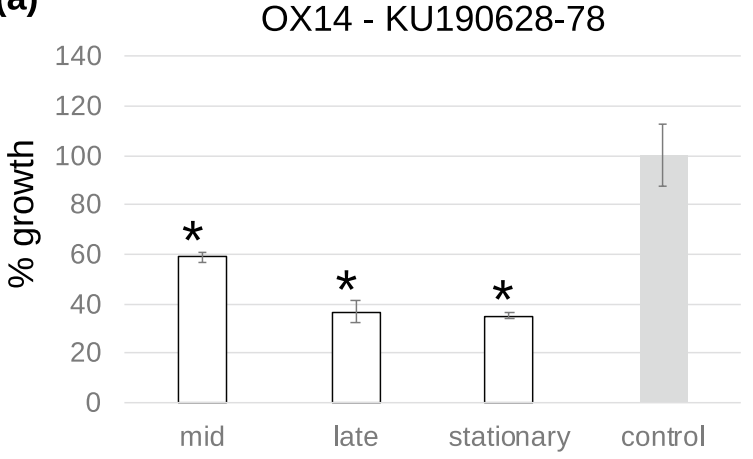

(b)

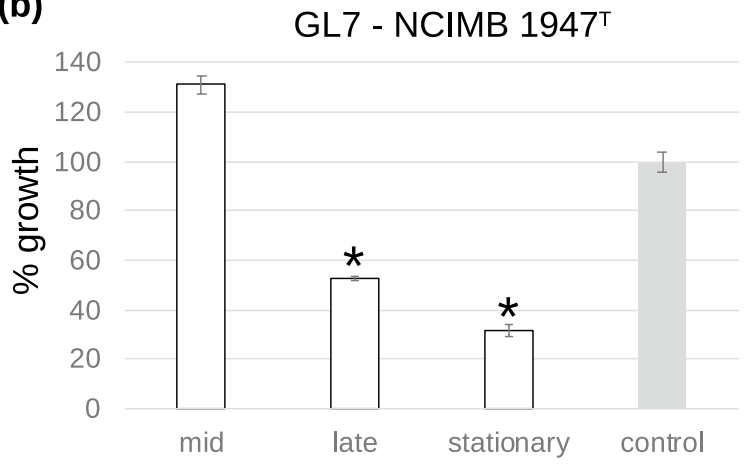

Figure 3. Antagonistic activity of bacterial culture supernatants against F. psychrophilum at various growth phases. Growth activities of (a) OX14 and (b) GL7 strains against F. psychrophilum KU190628-78 and NCIMB $1947^{\mathrm{T}}$, respectively, at mid and late exponential growth, and stationary phases. Growth of positive control, in the absence of the supernatant, is taken as $100 \%$ (OD values of positive controls are 0.2 for KU190628-78 and NCIMB $\left.1947^{\mathrm{T}}\right)$. Bars, standard deviation of triplicate samples. ${ }^{\star}$ Statistically significant decrease $(p<0.05)$.

(a)

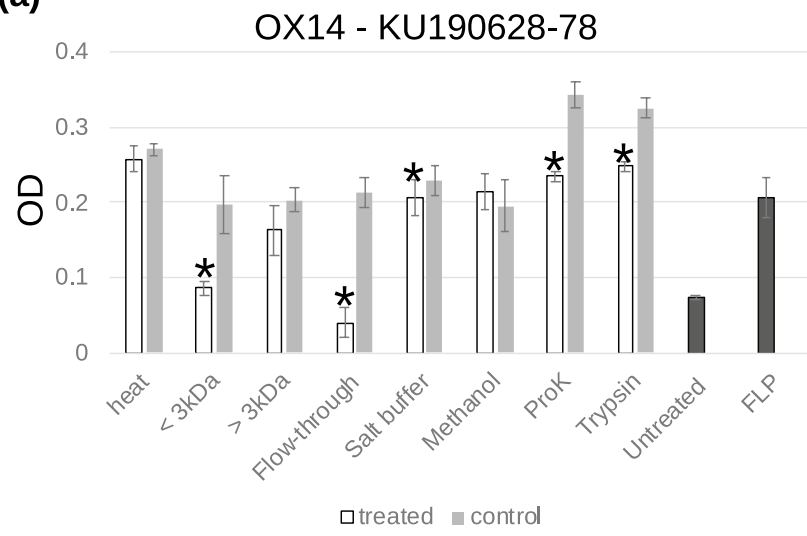

(b)
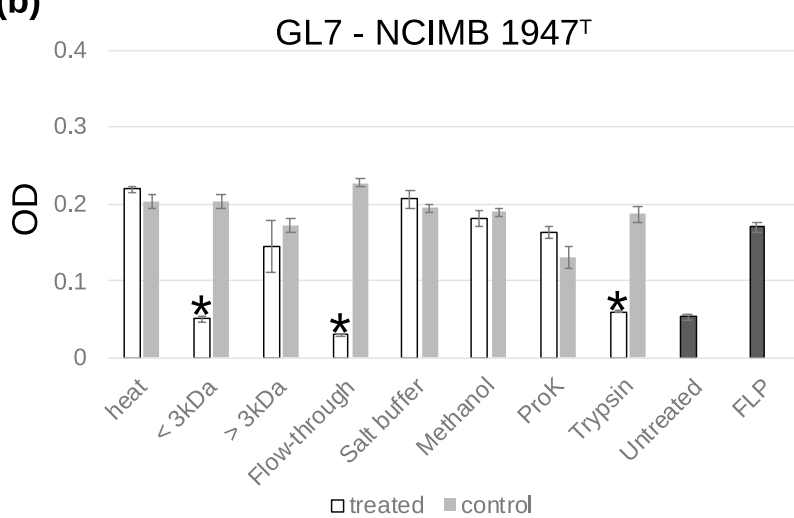

Figure 4. Antagonistic activity of heated, fractionated, and enzymatically digested bacterial culture supernatants against F. psychrophilum. Growth of (a) F. psychrophilum KU190628-78 and (b) F. psychrophilum NCIMB $1947^{\mathrm{T}}$ incubated with culture supernatant of strain OX14 and GL7, respectively (white bars). Growth of F. psychrophilum strains with FLP medium similarly treated is used as a positive control (gray bars). Flowthrough, flow-through from the anion exchange column. Salt buffer, eluate by high salt buffer. Methanol, eluate by methanol containing $1 \%$ formic acid. Growth of F. psychrophilum in untreated culture supernatant is presented as Untreated, and that in FLP medium is presented as FLP. Bars, standard deviation of the triplicates. ${ }^{\star}$ Statistically significant decrease.

possibility that the antagonistic ability of these strains may be associated with siderophore production (Supplementary Fig. S2 online).

Rheinheimera sp. generates hydrogen peroxide which oxidizes L-lysine to produce an antimicrobial macromolecule, L-lysin oxidase ${ }^{37}$. Therefore, production of hydrogen peroxide was also assayed by a modified Prussian blue method. Both of strain OX14 and GL7 were negative for hydrogen peroxide production.

The culture supernatant of strain OX14 inhibited the growth of F. psychrophilum KU190628-78 compared to the positive control experiment without culture supernatant. The growth inhibition was more effective during the late and stationary growth phases (35-37\% of the control; Fig. 3a) compared to the mid growth phase (59\% of the control; Fig. 3a). The culture supernatant of strain GL7 inhibited the growth of F. psychrophilum NCIMB 1947 ${ }^{\mathrm{T}}$, and was effective at the late exponential growth and stationary phases (32-53\% of the positive control; Fig. 3b).

Further analysis revealed that the antagonistic compounds produced by strains OX14 and GL7 were $<3 \mathrm{kDa}$ in size and lost their activities after heating at $121^{\circ} \mathrm{C}$ for $20 \mathrm{~min}$ (Fig. 4a,b). The antagonistic activity was lost in the supernatant of strain GL7 after proteinase K digestion (Fig. 4b). The culture supernatant of strain OX14 treated by proteinase $\mathrm{K}$ and trypsin inhibited the growth of F. psychrophilum compared to similarly treated control, but the activity was considered to be at least weakened by the treatment because OD values for these two were higher than those of F. psychrophilum in non-treated FLP medium (Fig. 4a). Fractions eluted with high salt buffer and methanol, which was considered to contain negatively charged proteins and small molecules, respectively, did not, or only slightly, inhibit the growth of F. psychrophilum (Fig. 4a,b). In contrast, the flow-through of the anion exchange column retained the antagonistic actions, suggesting that antagonistics were positively charged or neutral compounds at neutral $\mathrm{pH}$. In order to further confirm antagonistic activity, culture supernatants were diluted 5- and tenfold with FLP medium, and growth inhibition against F. psychrophilum strains was 


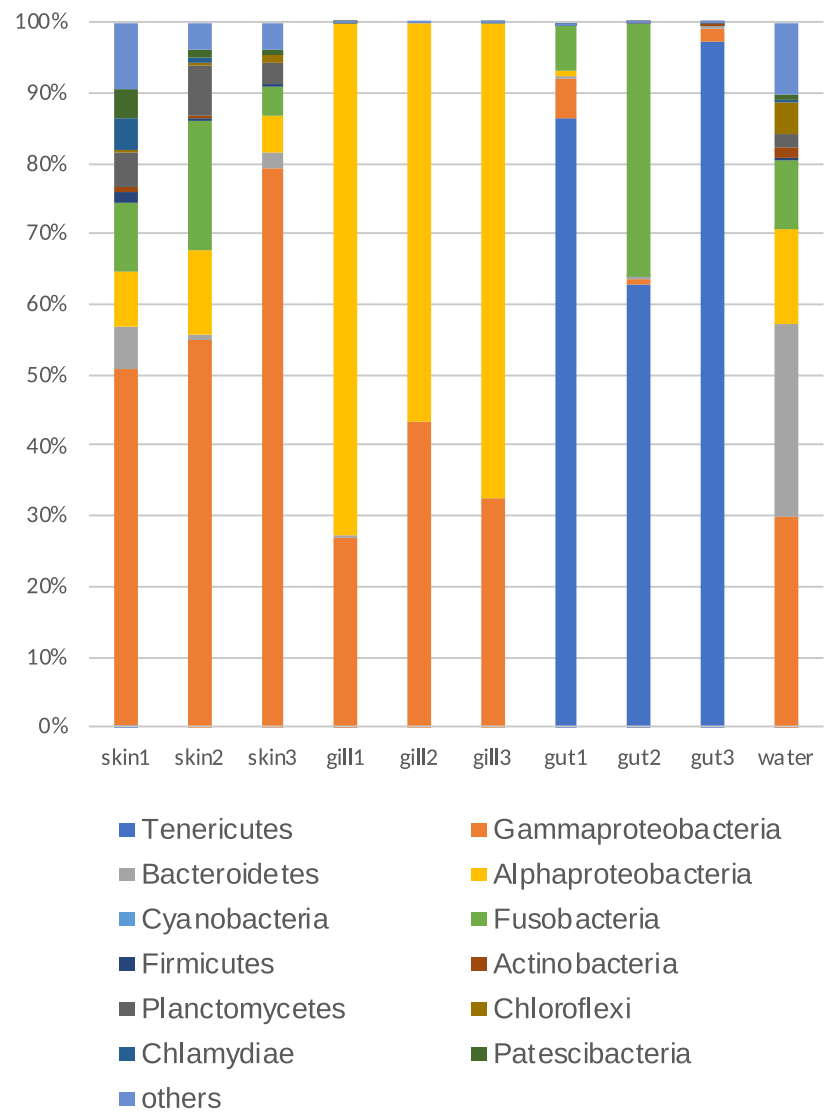

Figure 5. Relative abundance of phyla at sampling sites, and in individual fish, and rearing water.

examined. Five- and tenfold dilutions of the culture supernatant of strain OX14 did not inhibit the growth of $F$. psychrophilum KU190628-78. Five-fold dilution of the culture supernatant of strain GL7 inhibited the growth of F. psychrophilum NCIMB $1947^{\mathrm{T}}$ slightly (to $97.4 \%$ ), but no inhibition was observed when a tenfold dilution was used. In contrast, a tenfold concentrate of the culture supernatant of strain OX14 and GL7 obtained by freezedrying inhibited growth of F. psychrophilum to $3 \%$ of the control.

Microbial communities in rainbow trout and surrounding water. Supplementary Table S3 and Fig. S3 online show the general parameters of microbial communities characterized by 16S rRNA amplicon sequences. The number of reads that were finally used for analysis ranged between 30,203 and 44,849. The average numbers of OTUs (Sobs) per sample were 35, 69, and 380 for gill, the gut, and skin mucus, respectively, and 472 in the water sample. Skin and water shared 156 common OTU, whereas gill and the gut shared 1 and 4 OTU with water, respectively (Supplementary Fig. S4 online).

Figure 5 shows that Proteobacteria dominated (43\%-100\%) of the microbial communities in skin, gill, and water. Among the Proteobacteria, the genus Ralstonia (gamma-Proteobacteria) predominated the microbial communities in skin (32-68\%), whereas the genus Bosea (alpha-Proteobacteria) dominated those in the gill (57-74\%). The family Comamonadaceae was a major component of the Proteobacteria in water. The phyla, Fusobacteria and Planctomycete were also found in skin mucus microbiomes (Fig. 5). The dominant phylum (63-97\%) in the gut microbiome was Tenericutes (Fig. 5), which comprised one OTU belonging to the genus Mycoplasma. Others included Fusobacteria, i.e. Cetobacterium somerae at the species level, followed by Proteobacteria.

We compared our findings with two previous reports on the rainbow trout microbiome ${ }^{29,31}$. Proteobacteria was the dominant in skin microbiome of all of the studies. However, we found a difference in the major members next to Proteobacteria between the skin microbiomes in the present study and that described by Lowrey et al. ${ }^{29}$ and Terova et al. ${ }^{32}$. For example, next to Proteobacteria, Fusobacteria and Planctomycetes dominated in the present study (Fig. 5), whereas Bacteroidetes and Firmicutes were major phyla in Lowrey et al. ${ }^{29}$, and Tenericutes and Firmicutes in Terova et al. ${ }^{32}$. In contrast, the microbial communities in the gut were more similar, with the dominant family being Mycoplasmataceae (Tenericutes) in all of the studies.

Fraction of isolated strains in skin microbial communities of fish. Based on OTU clustering of $>99 \%$ sequence similarity, sequences related to the cultured isolates represented $4.95 \%, 61.41 \%, 0.01 \%$, and $10.03 \%$ of the microbial communities in skin, gill, the gut, and water, respectively (Table 2). On average, OTU similar to strain OX14 and GL7 accounted for $1.60 \%$ and $0.17 \%$ in skin microbial community, respectively. Sequences related to strain OX14 were the dominant in skin and gill microbial communities (Table 2). 


\begin{tabular}{|l|l|l|l|l|}
\hline Isolate & Skin-average $(\mathbf{n}=\mathbf{3})$ & Gill-average $(\mathbf{n}=\mathbf{3})$ & Gut-average $(\mathbf{n}=\mathbf{3})$ & Water $(\mathbf{n}=\mathbf{1})$ \\
\hline AG1 & 0.06 & 0.01 & 0.00 & 0.03 \\
\hline MN22 & 0.30 & 0.00 & 0.00 & 0.57 \\
\hline TLA9 & 0.16 & 0.00 & 0.00 & 0.18 \\
\hline TLA11 & 0.94 & 0.00 & 0.00 & 0.13 \\
\hline AG5 & 0.30 & 0.00 & 0.00 & 0.00 \\
\hline GL29 & 0.63 & 0.00 & 0.00 & 3.97 \\
\hline TLA8 & 0.02 & 0.00 & 0.00 & 0.10 \\
\hline MN10 & 0.06 & 0.00 & 0.00 & 0.08 \\
\hline OX16 & 0.04 & 0.00 & 0.00 & 0.42 \\
\hline GL27 & 0.01 & 0.00 & 0.00 & 0.70 \\
\hline GL7 & 0.17 & 0.00 & 0.01 & 0.11 \\
\hline MN5 & 0.00 & 0.00 & 0.00 & 0.14 \\
\hline OX15 & 0.00 & 0.00 & 0.00 & 0.00 \\
\hline OX14 & 1.60 & 61.40 & 0.00 & 0.04 \\
\hline OX5 & 0.07 & 0.00 & 0.00 & 0.02 \\
\hline MN24 & 0.00 & 0.00 & 0.00 & 0.00 \\
\hline OX13 & 0.12 & 0.00 & 0.00 & 0.00 \\
\hline OX11 & 0.43 & 0.00 & 0.00 & 3.35 \\
\hline OX32 & 0.00 & 0.00 & 0.00 & 0.00 \\
\hline MN7 & 0.02 & 0.00 & 0.00 & 0.04 \\
\hline Total & 4.95 & 61.41 & 0.01 & 10.03 \\
\hline & & & & \\
\hline
\end{tabular}

Table 2. Abundance (percentage) of each isolate in microbial communities of rainbow trout (in this study). Italics cells indicate sample with more than $0.01 \%$.

We also investigated whether our isolates were among the skin microbial communities of fish in other studies. We detected OTUs similar to strains OX14 and GL7 at rates of $0.01 \%$ in Lowrey et al. (2015) (Supplementary Table S4), but abundance of OTU having $>99 \%$ similarity with all of our isolates were lower $(0.02 \%)$ than that in our study (4.95\%). While OTUs similar to 9 out of 20 isolates were detected at $>0.01 \%$ in skin microbial communities of rainbow trout in Terova et al..$^{32}(0.54-0.88 \%$ in total), the proportions of sequences related to strain OX14 was low $(0.004 \%)$, and those related to GL7 was not detected (Supplementary Table S5). We detected OTU having $>99 \%$ similarity with all of our isolates except MN5 and MN10 in the skin microbial community of Atlantic salmon ${ }^{31}(0.39-10.79 \%$ in total, Supplementary Table S6). The ratios of OTUs similar to strains GL7 and OX14 in the skin microbial communities were $<0.09 \%$ and $<0.04 \%$, respectively.

\section{Discussion}

We isolated 174 bacterial strains from the skin mucus of rainbow trout spanning 18 genera. These strains were estimated to account for $4.95 \%$ of the total bacterial community in skin, although it should be noted that this can be an overestimation considering the low taxonomic resolution of short-reads ${ }^{38}$. These values were higher than those of brook charr (Salvelinus fontinalis) using two culture media $\left(0.03 \%-1.8 \%{ }^{15}\right)$. Spanggaard et al..$^{26}$ isolated 1,018 bacteria belonging to nine genera from skin, gill, and the gut of rainbow trout using TSA medium. As shown in Supplementary Table S2 online, multiple culture media used for isolation in the present study would have facilitated the isolation of diverse bacteria from the skin mucus of rainbow trout. Among isolates, strain GL29, which was most close to Acinetobacter tjernbergiae was most frequently obtained (Fig. 1), and this was one of the most frequently detected groups in the skin microbial community ( $0.63 \%$, Table 2$)$. Although this group was not detected in rainbow trout in Lowrey et al. ${ }^{29}$, it was detected in skin of rainbow trout in Terova et al. ${ }^{31}$, and was the most frequently found group in Atlantic salmon skin $(<7.06 \%$, Supplementary Table S6 online), suggesting that this group may be suitable to reside on skin of these fish. All of our strains grew on FLP medium, whereas several did not grow on OXOID CM3 and LB media (Supplementary Table S2 online). Hence, the FLP medium appears as a good alternative to the complex media commonly used for the isolation of relevant microorganisms in future studies. Our culture method expanded the range of culturable skin commensal bacteria that can be used to screen antagonistic bacteria against pathogens.

Strain OX14, which was closely related to Bosea lupini ${ }^{39}$, and strain GL7 that was most closely related to Flavobacterium tructae ${ }^{40}$ inhibited in vitro the growth of F. psychrophilum. They inhibited growth of F. psychrophilum to $>32 \%$ compared to the positive control, which was comparable to the growth inhibition by Pseudomonas sp. ${ }^{12}$, $\mathrm{M} 174^{10}, \mathrm{FF} 48^{13}$, and Enterobacter $\mathrm{C} 6-6^{17}$ against $F$. psychrophilum. To date, reported antagonistic bacteria against F. psychrophilum originating from fish skin were restricted to the genus Pseudomonas ${ }^{16}$ retrieved from rainbow trout and to five genera (Luteimonas, Microbacterium, Rhodococcus, Sphingopyxis, and Dietzia) retrieved from brook charr ${ }^{15}$. The present study added potentially antagonistic bacteria belonging to the genera, Flavobacterium and Bosea. Sequences related to strains OX14 and GL7 were comprising of 1.60 and $0.17 \%$ of the skin microbial community. Furthermore, sequences related to strain OX14 was the dominant in the gill microbial community 
(61.4\%) and also detected in rainbow trout used in previous studies ${ }^{29,31}$ although their population was lower compared to the present study. These strains may play a role in preventing F. psychrophilum at the surface of fish, the first line of defense, and could also be applicable in future biological treatment for fish diseases.

The production of siderophores ${ }^{10}$, bacteriocins ${ }^{41}$, lipoprotein ${ }^{17}$, hydrogen peroxide ${ }^{35}$, and blocking quorumsensing ${ }^{16}$ are considered as the mechanisms underlying the antagonistic ability of bacteria. Among these, GL7 produced siderophores, whereas OX14 was also weakly positive. Pérez -Pascual et al. ${ }^{42}$ recently reported that Flavobacterium sp. strain 4466 inhibited the growth of $F$. columnare although the mechanism of the inhibition is not still clear. The mechanism of antagonistic activity against fish pathogens is considered to be different between strains 4466 and GL7 because culture supernatant of strain GL7 was effective but not of strain 4466. Zhang et al. ${ }^{43}$ reported that several strains of the genus Bosea produce the quorum quenching enzyme, AHL lactonase. The size of the lactonase identified in Bosea was $30 \mathrm{kDa}^{43}$, which contradicts our finding that a $<3 \mathrm{kDa}$ molecule of the culture supernatants suppressed the growth of F. psychrophilum. Flow-through of culture supernatants from anion exchange columns retained antagonistic activity, suggesting that antagonistic compounds are small and positively charged or neutral compounds. Future study will focus on identifying antagonistic compounds.

Four F. psychrophilum strains responded differently to the test strains. For example, OX14 and GL7 respectively inhibited only the growth of F. psychrophilum KU190628-78 and NCIMB 1947T. These variable responses might be due to strain differences, which is also associated with difficulties developing vaccines ${ }^{7}$. The population structure of $F$. psychrophilum has been classified by multilocus sequence typing (MLST) ${ }^{44,45}$, and sequence types are highly correlated to the host fish species. In this study, we used four strains having distinct sequence types; the sequence types of NCIMB $1947^{\mathrm{T}}$ and SG950607 are CC-ST19 ${ }^{44}$ and CC-ST $10^{45}$, respectively, and those of KU190628-78 and KU190628-79 are CC-ST48 and CC-ST52, respectively (unpublished data). This suggests that the different reactions might be related to their sequence types. Although some studies have examined the effects of antagonistic bacteria against multiple strains or serotypes of $F$. psychrophilum ${ }^{10,12}$, the effects of antagonistic bacteria on distinct $F$. psychrophilum sequence types remain to be thoroughly elucidated. It would be important to examine the relationship between F. psychrophilum sequence types and their reactions to antagonistic strains, and the mechanisms for variable responses among the strains before practical application. We believe that strains OX14 and GL7 could be applicable to ayu and coho salmon, respectively, in biological treatment for fish disease. The skin microbiomes of ayu and coho salmon have not been described, but strain OX14 and GL7 could be detected in the skin of Atlantic salmon (Supplementary Table S6 online), suggesting that these can thrive on the skin of other types of fish.

Because strains OX14 and GL7 are likely to reside on skin rather than the gut (Table 2), it would not be appropriate to applying them as feed additives. Instead, manipulating the skin microbiome to increase their abundance is promising. The skin microbiome of fish is known to be more influenced by surrounding water compared to the gut microbiome ${ }^{32,46}$, which was also suggested by our data (Supplementary Fig. S3 online). We also found that skin microbiomes of rainbow trout were not so similar between the present findings and those of others ${ }^{29,31}$ compared to the gut microbiomes, although it should be also noted that many of other factors can also leads to different results ${ }^{47}$. Therefore, the microbial community of fish skin might be controlled by changing the microbial community in surrounding water in a closed system. However, simply adding bacterial strains to rearing tanks did not alter the skin microbial community of brook charr ${ }^{48}$. In future, it is necessary to establish a technology to manipulate fish skin microbiome.

In conclusion, we isolated 20 skin bacteria estimated to comprise $4.95 \%$ of the skin microbial community of rainbow trout, and obtained two strains with antagonistic action against F. psychrophilum. These skin bacteria might serve as the basis for a novel technology with which to prevent fish diseases.

\section{Methods}

Sample collection. Rainbow trout were obtained from Fukui-Chuo-Uoichi co., ltd and maintained at $16^{\circ} \mathrm{C}$ in 1,000-L tanks containing circulating groundwater at the Research Center for Marine Bioresources, Fukui Prefectural University, Japan for $>5$ months. Three healthy fish weighing 356-420 g were randomly sampled using a sterilized net, anesthetized with $0.05 \%$ 2-phenoxyethanol (Wako, Osaka, Japan), then gently rinsed with sterile water. Skin mucus was sampled using a sterile glass slide from the whole left side of the fish. The first gill arch was cut using sterilized scissors. The contents of the aseptically obtained gut were washed out by gently rinsing with sterilized water, and the guts were stored on ice. Microorganisms in rearing water were also collected by filtering $600 \mathrm{~mL}$ of water through Millipore Express PLUS filters with $0.22-\mu \mathrm{m}$ pores (Millipore Sigma Co., Ltd., Burlington, MA, USA). Samples were then brought to the laboratory on ice. Samples for DNA extraction were stored at $-80^{\circ} \mathrm{C}$. All experiments described above were conducted in compliance with the approved guidelines and regulations of Animal Care and Use Committee in Fukui Prefectural University. All procedures were approved by the same committee. Study design and reporting followed the ARRIVE guidelines.

Isolation of bacteria from skin mucus. We used nitrate mineral medium ${ }^{49}$ with vitamin solution (biotin $20 \mu \mathrm{g} \mathrm{L}{ }^{-1}$, folic acid $20 \mu \mathrm{g} \mathrm{L}{ }^{-1}$, thiamine- $\mathrm{HCl} 20 \mu \mathrm{g} \mathrm{L}^{-1}, \mathrm{~B} 121 \mu \mathrm{g} \mathrm{L} \mathrm{L}^{-1}$, calcium pantothenate $50 \mu \mathrm{g} \mathrm{L}{ }^{-1}$, riboflavin $50 \mu \mathrm{g} \mathrm{L}^{-1}$, nicotinamide $\left.50 \mu \mathrm{g} \mathrm{L} \mathrm{C}^{-1}\right)$ containing mannose $(0.5 \%)$, galactose $(0.5 \%)$, methanol $(1 \% \mathrm{v} / \mathrm{v})$, or methylamine $(0.5 \%)$ as carbon sources, or containing autoclaved skin mucus $(1 \% \mathrm{v} / \mathrm{v})$. We also used OXOID CM3 medium as a representative complex medium. Either calcium $(1.8 \mathrm{mM})$ or lanthanide $(30 \mu \mathrm{M})$ was added as a co-factor for methanol dehydrogenase ${ }^{50}$ in methanol medium. Plates contained agar, or the agar substitute Gelrite (CP Kelco, Atlanta, GA, USA) with methanol and methylamine media. Half of the skin mucus was diluted 4 to sixfold with $0.2 \%$ saline, and spread on the surface of the medium, then the plates were incubated at $16^{\circ} \mathrm{C}$. The other half of the skin mucus was stored at $-80^{\circ} \mathrm{C}$ for DNA extraction. After 5 days, colonies were subcultured on the same media. The original plates were further incubated and examined after 2 weeks of incubation. Newly 
emerged colonies were also picked and subcultured. One colony was transferred again onto the same medium to ensure a pure culture. At least 20 colonies were picked up from each plate (per carbon source) except for those of skin mucus that contained fewer colonies (Supplementary Table S1 online).

16S rRNA gene analysis of the isolates. Bacterial genomic DNA was extracted using phenol and chloroform as described by Cheng and Jiang ${ }^{51}$. The $16 \mathrm{~S}$ rRNA gene was amplified by PCR using TaKaRa Taq Hot Star Version (Takara Bio Inc., Kusatsu, Japan) and the primer sets, Eub8F (5'-AGAGTTTGATCCTGGCTCAG-3') and Univ1490R (5'-GGTTACCTTGTTACGACTT-3') for bacteria ${ }^{52}$. The PCR reaction (10 $\left.\mu \mathrm{L}\right)$ comprised 8 pmol of each primer, $1 \mu \mathrm{L}$ of $10 \times$ buffer, $0.8 \mu \mathrm{L}$ of dNTP mixture, $0.05 \mu \mathrm{L}$ of Takara Taq HS, and approximately $3 \mathrm{ng}$ of genomic DNA and proceeded under the following conditions: 30 cycles at $98^{\circ} \mathrm{C}$ for $10 \mathrm{~s}, 56^{\circ} \mathrm{C}$ for $30 \mathrm{~s}$, then $72^{\circ} \mathrm{C}$ for $1.5 \mathrm{~min}$. The PCR products were first sequenced with the universal primer $907 \mathrm{R}^{53}$. Sequence identities were calculated using ClustalX, and sequences with $>99 \%$ identity were considered as belonging to the same species and grouped accordingly. A representative strain was randomly selected from each group, and further analyzed. Almost the entire $16 \mathrm{~S}$ rRNA gene in each representative strain was sequenced and compared with the GenBank database using BLAST (http://www.ncbi.nlm.nih.gov/BLAST/).

Growth test with various media. The ability of isolates to grow on LB, OXOID CM3, FLP (complex media developed for F. psychrophilum ${ }^{54}$ ), and galactose containing media was assessed. Isolates were placed in $300 \mu \mathrm{L}$ of each medium in 96 well plates and incubated at $16^{\circ} \mathrm{C}$ for 2 weeks. Inoculated bacterial growth was periodically determined by measuring the OD at $630 \mathrm{~nm}$ using a Multiskan JX microplate reader (Thermo Labsystems Inc., Philadelphia PA, USA).

Screening bacteria antagonistic to Flavobacterium psychrophilum. The F. psychrophilum strains, NCIMB $1947^{\mathrm{T}}$ (isolated from O. kisutch), SG950607 (isolated from O. mykiss), KU190628-78 and KU19062879 (isolated from P. altivelis) were the target pathogens. The growth of F. psychrophilum strains in FPL medium containing one-fifth volume of culture supernatants of the test strains was measured using microplates. All test strains except M22 were cultured in FLP medium until the early stationary phase. Strain M22 was cultured with galactose containing medium due to low growth yield in FLP medium. Culture supernatant obtained by centrifugation at $13,000 \times \mathrm{g}$ for $6 \mathrm{~min}$, was passed through filters with $0.2-\mu \mathrm{m}$ pores. F. psychrophilum strains were precultured in test tubes containing FLP medium at $16^{\circ} \mathrm{C}$ for 2 days (OD reaches $0.3-0.4$ ). Microplate wells containing $50 \mu \mathrm{L}$ of supernatant and $10 \mu \mathrm{L}$ of $F$. psychrophilum culture in FLP medium (final volume, $250 \mu \mathrm{L}$ ) were incubated in triplicate at $16^{\circ} \mathrm{C}$ for 2 days. The negative and positive controls were FLP medium without and with F. psychrophilum strains, respectively. The turbidity in each well was measured at $660 \mathrm{~nm}$ using a Wallac1420 ARVO MX/Light microplate reader (PerkinElmer Life and Analytical Sciences Inc., Waltham, MA, USA).

Cross-streaking method. Cross-streaking method was used to further confirm the antagonistic activity of strains OX14 and GL7 to F. psychrophilum KU190628-78 and NCIMB $1947^{\mathrm{T}}$, respectively. For this, suspensions of the pathogens were streaked at right angles to the line of the cultures of strains OX14 and GL7 previously grown on FLP plates for 7 days, and incubated at $16^{\circ} \mathrm{C}$ for 2 days. Antagonism by strains OX14 and GL7 was indicated by an interruption in the growth of the pathogen.

Characterization of antagonistic compounds. Some microorganisms produce siderophores to capture iron from environments, and this is one mechanism through which antagonistic bacteria inhibit the growth of pathogens ${ }^{10,16}$. We assessed siderophore production using chrome azurol S (CAS) assays as described by Machuca and Milagres ${ }^{55}$ with modification. A hole in the FLP medium with a diameter of $3 \mathrm{~cm}$ was aseptically punched, and CAS-blue agar was poured into it. Test strains were inoculated across the border of the two media and the plates were incubated at $16^{\circ} \mathrm{C}$ for one week. The color change of the CAS-blue agar was considered as an indication of positive reaction.

Production of hydrogen peroxide was assayed by a modified Prussian blue method ${ }^{56}$ due the reactivity of the FLP medium with hexacyanoferrate(III). $1 \mathrm{~g}$ each of $\mathrm{FeCl}_{3} \cdot 6 \mathrm{H}_{2} \mathrm{O}$ and potassium hexacyanoferrate(III) was dissolved in separate $50 \mathrm{~mL}$ of water. These solutions $(50 \mu \mathrm{L})$ were added to $900 \mu \mathrm{L}$ of water. Then, test solution of $50 \mu \mathrm{L}$ was added to monitor the color change of the mixture after $5 \mathrm{~min}$. This modified method was as sensitive as the original Prussian blue agar method ( $>1 \mathrm{nmol}$ of hydrogen peroxide). Strains OX14 and GL7 were grown in galactose containing medium, and the culture supernatants were used as test solutions.

To further characterize antagonistic compounds produced by strains OX14 and GL7, we assayed the antagonistic action of culture supernatants sampled at mid and late exponential, and stationary phases as described above. Culture supernatants were also assessed after heating at $121^{\circ} \mathrm{C}$ for $20 \mathrm{~min}$, fractionation with Centriprep YM-3 (Merck KGaA, Darmstadt, Germany) to collect compounds of $<$ and $>3 \mathrm{kDa}$ and following proteinase $\mathrm{K}$ and trypsin digestion. These enzymes in $20 \mathrm{mM}$ Tris- $\mathrm{HCl}(\mathrm{pH} 8.0)$ were added to the culture supernatant at a final concentration of $0.1 \mathrm{mg} \mathrm{mL}^{-1}$ and incubated at $37^{\circ} \mathrm{C}$ for $3 \mathrm{~h}$. Culture supernatants $(6 \mathrm{~mL})$ were loaded onto an Oasis MAX cartridge $3 \mathrm{cc}$ (Waters Corp., Milford, MA, USA), and negatively charged proteins were eluted with $1 \mathrm{~mL}$ of high salt buffer (HEPES $50 \mathrm{mM}, 0.5 \mathrm{M} \mathrm{NaCl}, \mathrm{pH}$ 7.2). Other acidic small compounds were further eluted with $1 \mathrm{~mL}$ of methanol containing $1 \%$ formic acid ${ }^{57}$. For each treatment, FLP medium similarly treated was used as a positive control. Five- and tenfold-diluted supernatants as well as tenfold concentrate by freeze-drying were also assayed. 
Analysis of 16S rRNA amplicon sequences. We extracted DNA from skin mucus, gill, the gut (posterior gut) of rainbow trout, and the rearing water using the FastDNA SPIN Kit for Soil (MP Biomedicals Inc., Santa Ana, CA, USA) as described by the manufacturer. Gill samples were suspended in $0.2 \%$ saline, and homogenized using a sterile spatula. Large pieces of debris were removed by centrifugation at $300 \times \mathrm{g}$ for $1 \mathrm{~min}$, then microbial cells were collected by further centrifugation of the supernatant at $5000 \times \mathrm{g}$ for $5 \mathrm{~min}$. Microorganisms in the rearing water were resuspended in $5 \mathrm{~mL}$ of sterilized water, and collected by centrifugation at $5000 \times \mathrm{g}$ for $5 \mathrm{~min}$, then DNA was extracted. Archaea are minor components of the microbial community in fish $\mathrm{skin}^{58}$; thus, the V3-V4 region (approximately $450 \mathrm{bp}$ ) of the bacterial $16 \mathrm{~S}$ rRNA gene was amplified using PCR with primers 5' ACACTCTTTCCCTACACGACGCTCTTCCGATCT- NNNNN- fwd_primer-3' and 5'-GTGACTGGA GTTCAGACGTGTGCTCTTCCGATCT-NNNNN-rev_primer', where fwd_primer is $341 \mathrm{~F}^{59}$ and rev_primer is $805 \mathrm{R}^{59}$. PCR was performed with Good Taq (Intégrale Co., Ltd. Tokyo, Japan) under the following conditions: $95^{\circ} \mathrm{C}$ for $10 \mathrm{~min} ; 30 \mathrm{cycles}$ at $95^{\circ} \mathrm{C}$ for $30 \mathrm{~s}, 55^{\circ} \mathrm{C}$ for $30 \mathrm{~s}$, then $72^{\circ} \mathrm{C}$ for $40 \mathrm{~s}$. The PCR products were cleaned with AMPure XP (Beckman Coulter, Brea, CA) and used for indexing PCR. The PCR reaction $(10 \mu \mathrm{L})$ comprised 5 pmol of each primer, $1 \mu \mathrm{L}$ of $10 \times$ Ex buffer, $0.8 \mu \mathrm{L}$ of dNTP mixture, $0.1 \mu \mathrm{L}$ of TaKaRa Ex Taq HS (Takara Bio Inc., Kusatsu, Japan), and 10 ng of the purified PCR product and proceeded under the following conditions: $94{ }^{\circ} \mathrm{C}$ for $2 \mathrm{~min}, 10$ cycles at $94^{\circ} \mathrm{C}$ for $30 \mathrm{~s}, 60^{\circ} \mathrm{C}$ for $30 \mathrm{~s}$, and $72{ }^{\circ} \mathrm{C}$ for $30 \mathrm{~s}$, then $72{ }^{\circ} \mathrm{C}$ for $5 \mathrm{~min}$. After another round of AMPure XP (Beckman Coulter, Brea, CA) clean-up, multiplexed amplicons were sequenced with Illumina MiSeq (Illumina, CA, 300 bp paired-end reads) at Seibutsu Giken Co., Ltd (Kanagawa, Japan).

The sequences were analyzed using mothur ver. $43^{60}$ as previously described ${ }^{61}$ with the following modifications. Chimeric sequences were removed using VSEARCH ${ }^{62}$. Singleton was removed to reduce error ${ }^{63}$. After clustering sequences into operational taxonomic units (OTUs) using the OptiClust algorithm ${ }^{64}$, each unique OTU was categorized by a Bayesian classifier and on the Silva taxonomy SSU Ref NR99 release 132 datasets ${ }^{65}$ with a confidence threshold of $80 \%$. Taxonomic information of the OTUs ( $>97 \%$ sequence similarity) was obtained from the majority consensus taxonomies of the sequences within the OTU with a consensus confidence threshold of $80 \%$. We also conducted OTU clustering (>99\% sequence similarity) using Illumina sequencing reads and Sanger-based sequences of isolates to determine the abundance of isolates in the microbial communities. Data in Lowrey et al. ${ }^{29}$, Terova et al. ${ }^{31}$ and Webster et al. ${ }^{32}$ were also analyzed in this manner.

Statistical analysis. Differences between positive controls and test samples were calculated using Student's t tests ${ }^{66}$. Values with $\mathrm{p}<0.05$ were deemed statistically significant.

\section{Data availability}

The 16S rRNA gene sequences of the isolates determined herein were deposited in the DDBJ/GenBank/EMBL database under accession numbers LC566124 -LC566143. Other sequence data generated in this study were deposited in the NCBI Sequence Read Archive under BioProject ID PRJDB10074.

Received: 20 November 2020; Accepted: 24 March 2021

Published online: 06 April 2021

\section{References}

1. FAO. Fisheries and Aquaculture Department. The State of World Fisheries and Aquaculture 2016 204p (Food and Agriculture Organization of the United Nations, Rome, 2016).

2. Borg, A. F. Studies on Myxobacteria associated with diseases in salmonid fishes. Wildl. Dis. 8, 1-85 (1960).

3. Wakabayashi, H., Toyama, T. \& Iida, T. A study on serotyping of Cytophaga psychrophila isolated from fishes in Japan. Fish Pathol. 29, 101-104 (1994).

4. Lulijwa, R., Rupia, E. J. \& Alfaro, A. C. Antibiotic use in aquaculture, policies and regulation, health and environmental risks: A review of the top 15 major producers. Rev. Aquac. 12, 640-663 (2020).

5. World Health Organization. Ten threats to global health in 2019. https://www.who.int/news-room/spotlight/ten-threats-to-globalhealth-in-2019 (2019)

6. White, A. \& Hughes, J. M. Critical importance of a one health approach to antimicrobial resistance. EcoHealth 16, 404-409 (2019).

7. Gómez, E., Mendez, J., Cascales, D. \& Guijarro, J. A. Flavobacterium psychrophilum vaccine development: A difficult task. Microb. Biotechnol. 7, 414-423 (2014).

8. Pérez-Sánchez, T., Mora-Sánchez, B. \& Balcázar, J. L. Biological approaches for disease control in aquaculture: Advantages, limitations and challenges. Trends Microbiol. 26, 896-903 (2018).

9. Robertson, P. A., O’Dowd, C., Burrells, C., Williams, P. \& Austin, B. Use of Carnobacterium sp. as a probiotic for Atlantic salmon (Salmo salar L.) and rainbow trout (Oncorhynchus mykiss, Walbaum). Aquaculture 185, 235-243 (2000).

10. Korkea-aho, T. L., Heikkinen, J., Thompson, K. D., von Wright, A. \& Austin, B. Pseudomonas sp. M174 inhibits the fish pathogen Flavobacterium psychrophilum. J. Appl. Microbiol. 111, 266-277 (2011).

11. Korkea-aho, T. L. et al. Pseudomonas M162 confers protection against rainbow trout fry syndrome by stimulating immunity. J. Appl. Microbiol. 113, 24-35 (2012).

12. Ström-Bestor, M. \& Wiklund, T. Inhibitory activity of Pseudomonas sp. on Flavobacterium psychrophilum, in vitro. J. Fish Dis. 34, 255-264 (2011).

13. De la Fuente, M., Vidal, J. M., Miranda, C. D., González, G. \& Urrutia, H. Inhibition of Flavobacterium psychrophilum biofilm formation using a biofilm of the antagonist Pseudomonas fluorescens FF48. Springerplus 2, 176 (2013).

14. Itoh, K., Nakai, Y., Ineno, T., Taguchi, T. \& Maeda, M. Antagonistic activities of bacteria against the pathogen of cold-water disease, Flavobacterium psychrophilum. Oceanogr. Jpn. 15, 417-423 (2006).

15. Boutin, S., Bernatchez, L., Audet, C. \& Derôme, N. Antagonistic effect of indigenous skin bacteria of brook charr (Salvelinus fontinalis) against Flavobacterium columnare and F. psychrophilum. Vet. Microbiol. 155, 355-361 (2012).

16. De la Fuente, M. et al. Growth inhibition of bacterial fish pathogens and quorum-sensing blocking by bacteria recovered from Chilean salmonid farms. J. Aquat. Anim. Health 27, 112-122 (2015).

17. Schubiger, C. et al. Entericidin is required for a probiotic treatment (Enterobacter sp strain C6-6) to protect trout from cold-water disease challenge. Appl. Environ. Microbiol. 81, 658-665 (2015).

18. Irianto, A. \& Austin, B. Probiotics in aquaculture. J. Fish Dis. 25, 633-642 (2002). 
19. Nakashima, K. et al. Chitin-based barrier immunity and its loss predated mucus-colonization by indigenous gut microbiota. Nat. Commun. 9, 3402 (2018).

20. Miwa, S. \& Nakayasu, C. Pathogenesis of experimentally induced bacterial cold water disease in ayu Plecoglossus altivelis. Dis. Aquat. Org. 67, 93-104 (2005).

21. Lagier, J. C. et al. Microbial culturomics: Paradigm shift in the human gut microbiome study. Clin. Microbiol. Infect. 18, 1185-1193 (2012).

22. Horsley, R. W. The bacterial flora of the Atlantic Salmon (Salmo salar L.) in relation to its environment. J. Appl. Bacteriol. 36, 377-386 (1973).

23. Westerdahl, A., Olsson, J. C., Kjelleberg, S. \& Conway, P. L. Isolation and characterization of turbot (Scophthalmus maximus)associated bacteria with inhibitory effects against Vibrio anguillarum. Appl. Environ. Microbiol. 57, 2223-2228 (1991).

24. Smith, P. \& Davey, S. Evidence for the competitive exclusion of Aeromonas salmonicida from fish with stress inducible furunculosis by a fluorescent pseudomonad. J. Fish Dis. 16, 521-524 (1993).

25. Schmidt, A. S., Bruun, M. S., Dalsgaard, I., Pedersen, K. \& Larsen, J. L. Occurrence of antimicrobial resistance in fish-pathogenic and environmental bacteria associated with four Danish rainbow trout farms. Appl. Environ. Microbiol. 66, 4908-4915 (2000).

26. Spanggaard, B. et al. The probiotic potential against vibriosis of the indigenous microflora of rainbow trout. Environ. Microbiol. 3, 755-765 (2001).

27. Arias, C. R., Koenders, K. \& Larsen, A. M. Predominant bacteria associated with red snapper from the Northern Gulf of Mexico. J. Aquat. Anim. Health 25, 281-289 (2013).

28. Musharrafieh, R., Tacchi, L., Trujeque, J., LaPatra, S. \& Salinas, I. Staphylococcus warneri, a resident skin commensal of rainbow trout (Oncorhynchus mykiss) with pathobiont characteristics. Vet. Microbiol. 169, 80-88 (2014).

29. Lowrey, L., Woodhams, D. C., Tacchi, L. \& Salinas, I. Topographical mapping of the rainbow trout (Oncorhynchus mykiss) microbiome reveals a diverse bacterial community with antifungal properties in the skin. Appl. Environ. Microbiol. 81, 6915-6925 (2015).

30. Kluga, A., Kacaniova, M., Kántor, A., Kaspars, K. \& Terentjeva, M. Identification of microflora of freshwater fish caught in the Driksna river and pond in Latvia. Foodbalt 2017, 164-168 (2017).

31. Terova, G. et al. Effects of full replacement of dietary fishmeal with insect meal from Tenebrio molitor on rainbow trout gut and skin microbiota. J. Anim. Sci. Biotechnol. 12, 30 (2021).

32. Webster, T. M. U., Consuegra, S., Hitchings, M. \& Garcia de Leaniz, C. Interpopulation variation in the Atlantic salmon microbiome reflects environmental and genetic diversity. Appl. Environ. Microbiol. 84, e00691 (2018).

33. Dash, S., Das, S. K., Samal, J. \& Thatoi, H. N. Epidermal mucus, a major determinant in fish health: A review. Ind. J. Vet. Res. 19, 72-81 (2018).

34. Boutin, S., Bernatchez, L., Audet, C. \& Derôme, N. Network analysis highlights complex interactions between pathogen host and commensal microbiota. PLoS ONE 8, e84772 (2013).

35. Milijašević-Marčić, S. et al. Antagonistic potential of Bacillus spp isolates against bacterial pathogens of tomato and fungal pathogen of pepper. Pestic. Phytomed. 33, 9-18 (2018).

36. Yekkour, A. et al. Characterization and antagonistic properties of Streptomyces strains isolated from Saharan soils, and evaluation of their ability to control seedling blight of barley caused by Fusarium culmorum. Lett. Appl. Microbiol. 55, 427-435 (2012).

37. Chen, W. M., Lin, C. Y. \& Sheu, S. Y. Investigating antimicrobial activity in Rheinheimera sp. due to hydrogen peroxide generated by L-lysine oxidase activity. Enzyme Microb. Technol. 46, 487-493 (2010).

38. Johnson, J. S. et al. Evaluation of $16 \mathrm{~S}$ rRNA gene sequencing for species and strain-level microbiome analysis. Nat. Commun. 10, $5029(2019)$.

39. De Meyer, S. E. \& Willems, A. Multilocus sequence analysis of Bosea species and description of Bosea lupini sp. nov., Bosea lathyri sp. nov. and Bosea robiniae sp. nov., isolated from legumes. Int. J. Syst. Evol. Microbiol. 62, 2505-2510 (2012).

40. Zamora, L. et al. Flavobacterium tructae sp. nov. and Flavobacterium piscis sp. nov., isolated from farmed rainbow trout (Oncorhynchus mykiss). Int. J. Syst. Evol. Microbiol. 64, 392-399 (2014).

41. Stoffels, G., Nes, I. F. \& Guðmundsdóttir, Á. Isolation and properties of a bacteriocin-producing Carnobacterium piscicola isolated from fish. J. Appl. Bacteriol. 73, 309-316 (1992).

42. Pérez-Pascual, D. et al. Gnotobiotic rainbow trout (Oncorhynchus mykiss) model reveals endogenous bacteria that protect against Flavobacterium columnare infection. PLoS Pathog 17, e1009302 (2021).

43. Zhang, J. et al. AidB, a novel thermostable N-acylhomoserine lactonase from the bacterium Bosea sp. Appl. Environ. Microbiol. 85, e02065-e2119 (2019).

44. Nicolas, P. et al. Population structure of the fish-pathogenic bacterium Flavobacterium psychrophilum. Appl. Environ. Microbiol. 74, 3702-3709 (2008).

45. Fujiwara-Nagata, E. et al. Population structure of the fish pathogen Flavobacterium psychrophilum at whole-country and model river levels in Japan. Vet. Res. 44, 34 (2013).

46. Sylvain, F. É. et al. Fish skin and gut microbiomes show contrasting signatures of host species and habitat. Appl. Environ. Microbiol. 86, e0078920. https://doi.org/10.1128/aem.00789-20 (2020).

47. Manzoor, S. S., Doedens, A. \& Burns, M. B. The promise and challenge of cancermicrobiome research. Genome Biol. 21, 131 (2020).

48. Boutin, S., Audet, C. \& Derôme, N. Probiotic treatment by indigenous bacteria decreases mortality without disturbing the natural microbiota of Salvelinus fontinalis. Can. J. Microbiol. 59, 662-670 (2013).

49. Bowman, J. P. The methanotrophs-the families methylococcaceae and methylocystaceae. In The Prokaryotes Vol. 5 (eds Dworkin, M. et al.) 266-289 (Springer, Berlin, 2006).

50. Nakagawa, T. et al. A catalytic role of XoxF1 as $\mathrm{La}^{3+}$-dependent methanol dehydrogenase in Methylobacterium extorquens strain AM1. PLoS ONE 7, e50480 (2012).

51. Cheng, H. \& Jiang, N. Extremely rapid extraction of DNA from bacteria and yeasts. Biotechnol. Lett. 28, 55-59 (2006).

52. Weisburg, W. G., Barns, S. M., Pelletier, D. A. \& Lane, D. J. 16 S ribosomal DNA amplification for phylogenetic study. J. Bacteriol. 173, 697-703 (1991).

53. Lane, D. J. 16S/23S rRNA sequencing. In Nucleic Acid Techniques in Bacterial Systematics (eds Stackebrandt, E. \& Goodfellow, M.) 115-147 (Wiley, New York, 1991).

54. Cepeda, C., García-Márquez, S. \& Santos, Y. Improved growth of Flavobacterium psychrophilum using a new culture medium. Aquaculture 238, 75-82 (2004).

55. Machuca, Á. \& Milagres, A. M. Use of CAS-agar plate modified to study the effect of different variables on the siderophore production by Aspergillus. Lett. Appl. Microbiol. 36, 177-181 (2003).

56. Saito, M., Seki, M., Iida, K., Nakayama, H. \& Yoshida, S. A novel agar medium to detect hydrogen peroxide-producing bacteria based on the prussian blue-forming reaction. Microbiol. Immunol. 51, 889-892 (2007).

57. Fontanals, N., Borrull, F. \& Marcé, R. M. Mixed-mode ion-exchange polymeric sorbents in environmental analysis. J. Chromatogr. A 1609, 460531 (2020).

58. Chiarello, M. et al. Skin microbiome of coral reef fish is highly variable and driven by host phylogeny and diet. Microbiome 6, 147 (2018).

59. Herlemann, D. P. R. et al. Transitions in bacterial communities along the $2000 \mathrm{~km}$ salinity gradient of the Baltic Sea. ISME J. 5, 1571-1579 (2011). 
60. Schloss, P. D. et al. Introducing mothur: Open-source, platform-independent, community-supported software for describing and comparing microbial communities. Appl. Environ. Microbiol. 75, 7537-7541 (2009).

61. Katayama, T. et al. Changes in microbial communities associated with gas hydrates in subseafloor sediments from the Nankai Trough. FEMS Microbiol. Ecol. 92, fiw093 (2016).

62. Rognes, T., Flouri, T., Nichols, B., Quince, C. \& Mahé, F. VSEARCH: A versatile open 2source tool for metagenomics. PeerJ 4 , e2584 (2016).

63. Reeder, J. \& Knight, R. The 'rare biosphere': A reality check. Nat. Methods 6, 636-637 (2009).

64. Westcott, S. L. \& Schloss, P. D. OptiClust, an improved method for assigning amplicon-based sequence data to operational taxonomic units. $m$ Sphere 2, e00073-17 (2017).

65. Quast, C. et al. The SILVA ribosomal RNA gene database project: improved data processing and web-based tools. Opens external link in new window. Nucleic Acids Res. 41, D590-D596 (2013).

66. Student. The probable error of a mean. Biometrika 6, 1-25.

\title{
Acknowledgements
}

We thank N. Kitamura, Y. Fukuda, and Dr. C. Wu, AIST for their help in experiments. The strain SG950607 was kindly gifted by the Shiga Prefectural Fisheries Experimental Station.

\section{Author contributions}

M.T. isolated bacterial strains and wrote the main manuscript text, E.F.N. conducted antagonistic activity assay, T.K. analyzed amplicon sequence data, and H.S. provided resource. All authors reviewed and edited the manuscript.

\section{Competing interests}

The authors declare no competing interests.

\section{Additional information}

Supplementary Information The online version contains supplementary material available at https:/doi.org/ 10.1038/s41598-021-87167-1.

Correspondence and requests for materials should be addressed to M.T.

Reprints and permissions information is available at www.nature.com/reprints.

Publisher's note Springer Nature remains neutral with regard to jurisdictional claims in published maps and institutional affiliations.

\begin{abstract}
(c) (i) Open Access This article is licensed under a Creative Commons Attribution 4.0 International cc. License, which permits use, sharing, adaptation, distribution and reproduction in any medium or format, as long as you give appropriate credit to the original author(s) and the source, provide a link to the Creative Commons licence, and indicate if changes were made. The images or other third party material in this article are included in the article's Creative Commons licence, unless indicated otherwise in a credit line to the material. If material is not included in the article's Creative Commons licence and your intended use is not permitted by statutory regulation or exceeds the permitted use, you will need to obtain permission directly from the copyright holder. To view a copy of this licence, visit http://creativecommons.org/licenses/by/4.0/.
\end{abstract}

(c) The Author(s) 2021 